\title{
Critical COVID-19 Pneumonia with Acute Respiratory Failure in a Healthy 12-year-old Girl
}

\author{
Dolezalova Karolina ${ }^{1 *}$, Cabelova Tamara ${ }^{1}$, Hecht Tomas ${ }^{1}$, Heinige Pavel ${ }^{2}$ \\ ${ }^{1}$ Department of Paediatrics, First Faculty of Medicine, Charles University, Thomayer University Hospital \\ ${ }^{2}$ Clinic of Paediatric Surgery and Traumatology, Third Faculty of Medicine, Thomayer University Hospital \\ *Corresponding author email: karolina.dolezalova@ftn.cz \\ Received: 07 July 2021 / Revised: 04 September 2021 / Accepted: 06 September 2021 / Published: 07 September 2021
}

\begin{abstract}
The authors present a case report of an otherwise healthy, fully immunized 12-year-old girl who had a critical course of COVID-19 infection with acute respiratory failure. The epidemiologic history was positive for COVID-19, and she tested PCR positive resulting from a nasopharyngeal swab. She was presented with fever and cough to a regional pediatric department, and she was immediately intubated and transferred to a pediatric ICU in a University Hospital. CT of the thorax revealed bilateral consolidation with the tree-in-bud signs. Her condition required artificial ventilation support for 13 days. Remdesivir, pronation, high dose Ascorbic acid with Thiamine, and combined antimicrobial therapy were successfully used. The patient made a full clinical recovery. This case report is unique because of a very scarce critical course of COVID-19 infection in children. It demonstrates the successful use of a combined therapeutic approach with artificial ventilation, pronation, Remdesivir, and combined antimicrobial therapy. Clinical symptoms, laboratory results, imaging methods, and therapeutic attitude are mentioned to share our experience with the medical community.
\end{abstract}

Keywords: COVID-19, Children, Acute Respiratory Failure

\section{Introduction}

\section{$1.1 \quad$ Epidemiology}

Coronavirus disease 2019 (COVID-19), caused by severe acute respiratory syndrome coronavirus 2 (SARSCoV-2), originated in Wuhan and spread globally at a rapid pace causing 4,5 million deaths worldwide as of August 2021 [1]. Thus, the Czech Republic were in a critical epidemiological situation leading to the highest mortality worldwide, with actual evidence of 1.68 million infected patients and 30406 deaths [2]. Epidemiological evidence indicates that children are less likely to develop severe COVID-19 than adults. A prognosis for infected children is typically good with their "trained immunity" as a potential explanation. However, they only represent about 1-2 \% of the total COVID-19 burden. Clinical features of acute COVID-19 infection in children can be classified according to severity (asymptomatic, mild, moderate, severe, critical). Severe symptoms were reported in $5.9 \%$ of the children [3].

\subsection{Symptomatology}

The most prevalent symptom in the children infected with SARS-CoV-2 virus reported in a system analysis by Souza et al. [4] was fever (47.5\% of the cases) followed by cough ( $41.5 \%)$, nasal symptoms $(11.2 \%)$, diarrhoea $(8.1 \%)$, nausea/vomiting $(7.1 \%)$, fatigue $(5.0 \%)$, and respiratory distress $(3.5 \%)$. One hundred forty-five $(36.9 \%)$ children were diagnosed with pneumonia, and forty-three $(10.9 \%)$ upper airway infections were reported. Amongst the most common clinical signs described were pharyngeal erythema $(20.6 \%)$, tachycardia $(18.6 \%)$, and tachypnea $(13.4 \%)$ on admission. 


\subsection{Imaging Methods}

Typical radiological changes of COVID-19 pneumonia in children can be detected on X-ray or CT scans. According to Palabyniak et al. [5], X-ray abnormalities can be unilateral (55\%) and bilateral (45\%). Lesions are single or multiple with no side preference. Of the lesions, 13 were single $(48 \%)$ and 14 were numerous (52\%). The lower zones were the most commonly affected areas. A CT scan is a better option for the visualization of lung pathologies due to COVID-19. The most prevalent abnormalities reported on CT scans were ground-glass opacities, patchy shadows, and consolidations. Typical laboratory abnormalities were leucopenia or leucocytosis, mild elevation of CRP and PCT, and liver enzymes.

\section{Case Report}

We present a case of a 12-year-old otherwise healthy girl with overweight (BMI 24, 95th percentile). Her illness developed on the $12^{\text {th }}$ day after contacting with COVID-19 positive brother, the first signs being headache and fever [6]. After three more days, a maculopapular rash appeared on her forearms, palms and feet, and later changed its appearance into a large macular rash. Because of worsening dyspnea, she was brought into an emergency room of a district hospital on the $5^{\text {th }}$ day. Immediately, signs of respiratory distress were observed. Despite intensive oxygen therapy by semi-open mask with dosage $25 \mathrm{I} \mathrm{O}_{2} / \mathrm{min}$, her $\mathrm{SatO}_{2}$ levels were $85-92 \%$. Her heart rate was $150 / \mathrm{min}$ and tachypnea reaching $80 / \mathrm{min}$. Silent chest was the main finding in chest auscultation. To achieve improvement, she was acutely intubated and transferred to the Paediatric ICU of Thomayer University Hospital. On admission, she needed an aggressive ventilation setting: $\mathrm{FiO}_{2}$ (Inhaled Oxygen Fraction) being 1.0, PIP (peak inspiratory pressure) $35 \mathrm{cmH}_{2} \mathrm{O}$, she was also hypotensive $88 / 70$ torr with signs of peripheral vasoconstriction, heart rate at $78 / \mathrm{min}$. PCR test for COVID-19 was positive on admission, but so were Anti-SARS-CoV-2 antibodies. CRP was elevated $(115 \mathrm{mg} / \mathrm{l})$ despite PCT being negative. Chest X-ray revealed patchy opacities located in the right hemithorax. Figure 1 represents a Comparison of chest X-ray on the day of admission and 10 days after the admission. On admission: Pulmonary infiltration in right middle and upper lobe, smaller infiltration in the left lower lobe. Regression of infiltration on the $10^{\text {th }}$ day.

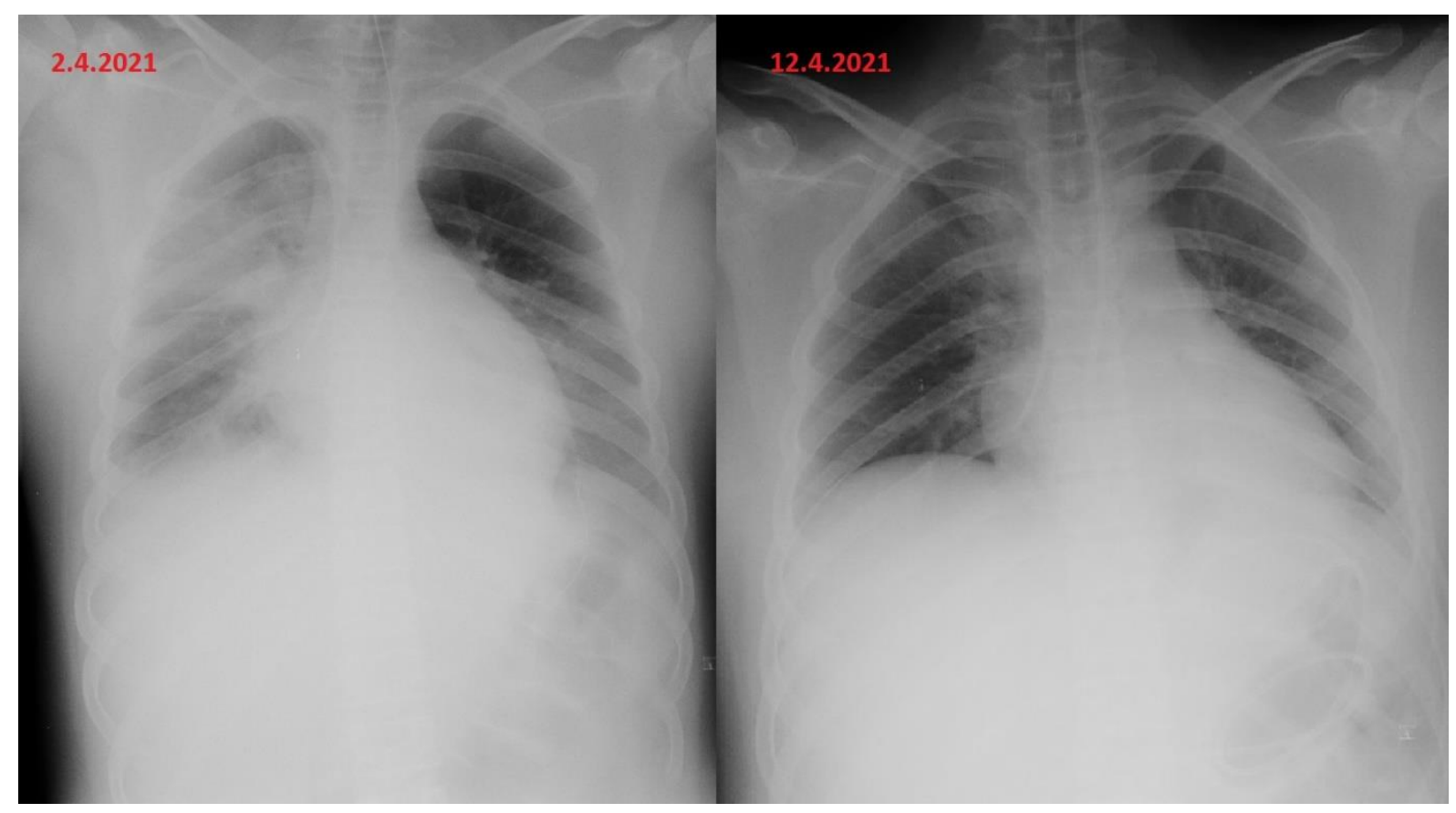

Figure 1: Comparison of chest X-ray on admission and after 10 days. On admission: Pulmonary infiltration in right middle and upper lobe, smaller infiltration in the left lower lobe. Regression of infiltration on the $10^{\text {th }}$ day. 
Dolezalova et al., Int. Ann. Sci.; Vol. 11, Issue 1, pp: 3-9, 2021

Chest ultrasound also confirmed bilateral fluidothorax and basal pulmonary condensation. In her arterial blood samples, signs of partial hypoxemic respiratory failure were present $\left(\mathrm{pO}_{2} 19,6 \mathrm{kPa}\right.$ and $\left.\mathrm{pCO}_{2}: 4,8 \mathrm{kPa}\right)$. These values were measured while using $\mathrm{FiO}_{2}$ 1.0, so the PFi $\left(\mathrm{PAO}_{2}\right.$ - Partial Artery Pressure of Oxygen $/ \mathrm{FiO}_{2}$ index) was $147 \mathrm{mmHg}$. Arterial hypotension was observed and treated by continuous norepinephrine. Medication and the aggressive ventilation regimen led to stabilizing the patient during the next two hours. However, arterial blood pressure increased up to $120 / 70 \mathrm{mmHg}$, so we decreased $\mathrm{FiO}_{2}$ down to 0.65 .

A further laboratory examination revealed high D-dimers prolonged aPTT. Troponin T and NTproBNP were regular. During ECHO examination, lowered EF (ejection fraction) was found so we added dobutamine to circulatory support.

Despite signs of RDS (respiratory distress syndrome), we took into account the symptoms of MIS-C syndrome (Multisystem Inflammatory Syndrome in Children) mentioned above (rash, decreased EF of the left ventricle, and the positive antibodies against SARS-CoV-2). Thus, we started with an immunosuppression protocol used in MIS-C patients, which was quickly abandoned soon after the final diagnose of COVID-19 pneumonia had been settled.

Concerning pharmacotherapy, remdesivir and high dose Ascorbic acid protocol with Thiamine were introduced. Flexible bronchoscopy was performed with physiological appearance of airways. We obtained negative microbial cultivation and positive COVID-19 PCR confirmation from bronchoalveolar lavage. CT scan of the lung revealed bilateral consolidation with the tree-in-bud signs but without ground-glass opacities. Figure 2 shows Axial CT of the thorax. Comparison of chest CT 4 days after admission where are bilateral patchy shadows and consolidations with small fluidothorax $(13 \mathrm{~mm}$ on right side, $7 \mathrm{~mm}$ on left side) and 18 days after admission with complete regression of inflammatory changes.

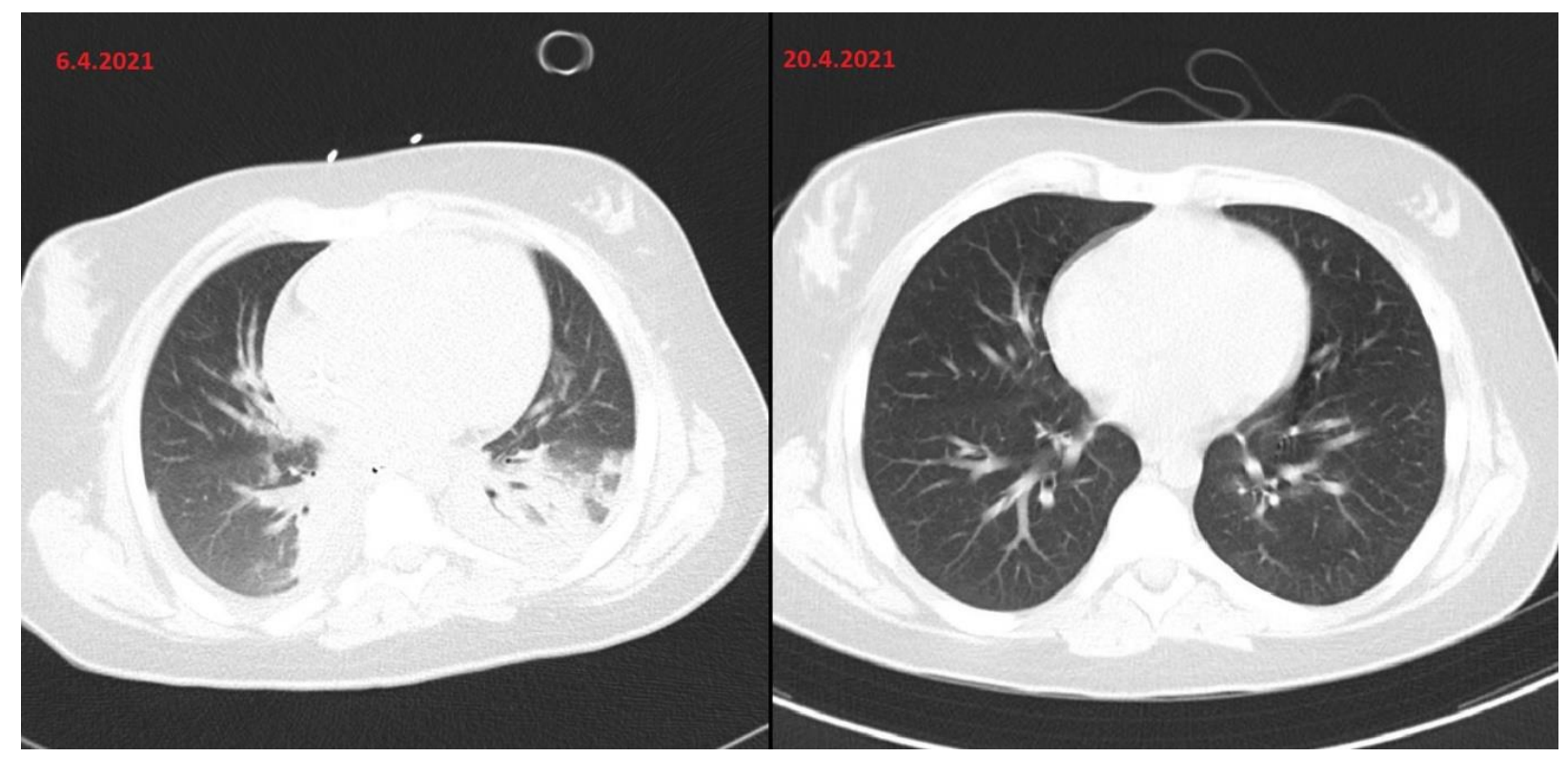

Figure 2: Axial CT of the thorax. Comparison of chest CT 4 days after and 18 days after admission with complete regression of inflammatory changes. 
Figure 3 shows CT of the thorax, anteroposterior view (2D reconstruction). 4 days after admission, bilateral consolidation with air bronchogram in posterior segments of right upper lobe and left lower lobe. 18 days after admission, the lung parenchyma is physiological.
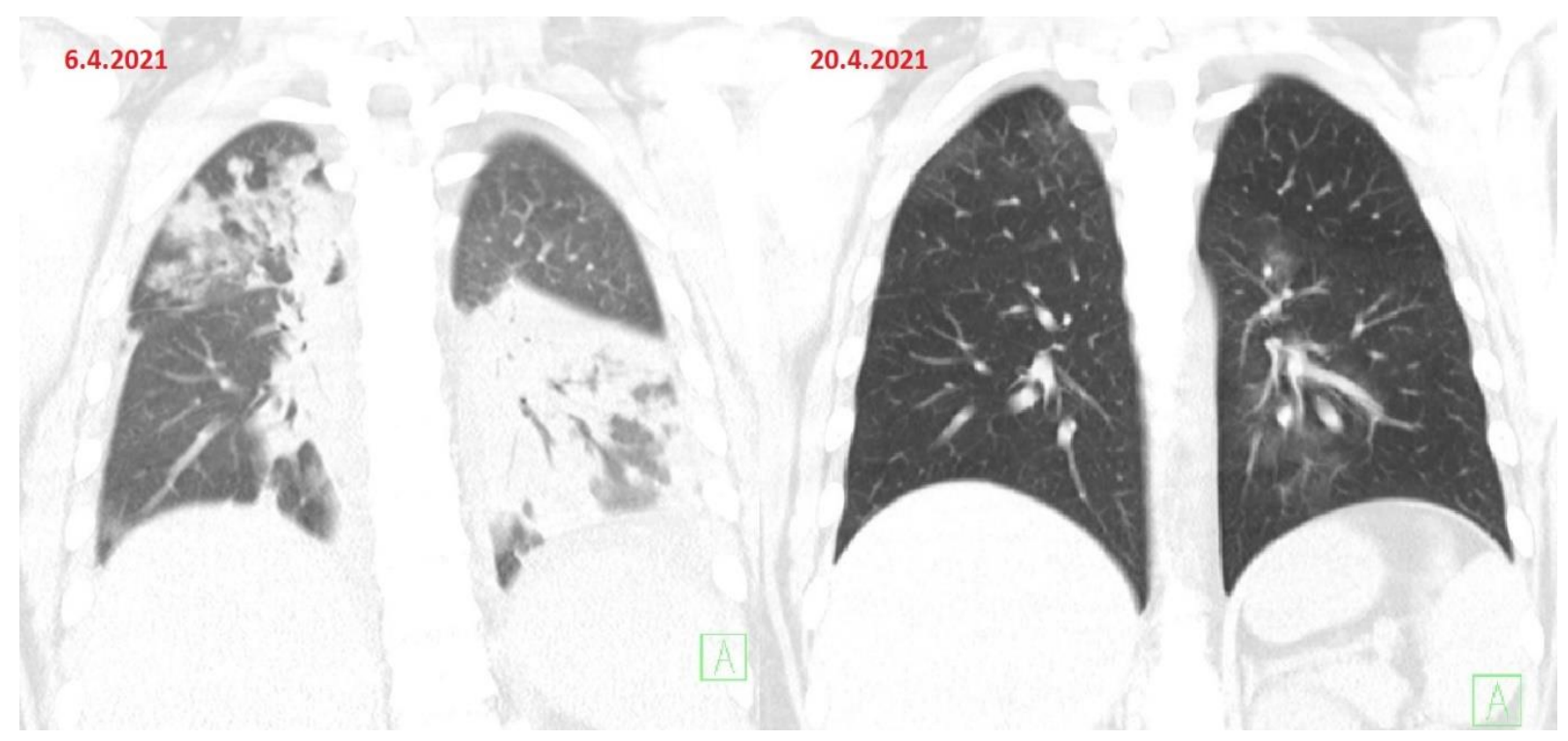

Figure 3: $C T$ of the thorax, anteroposterior view (2D reconstruction). Comparison of CT 4 days after admission and 18 days after admission, the lung parenchyma is physiological.

Exantema presented the first three days after admission spontaneously disappeared. Skin peeling on hand and feet, which is typical in MIS-C syndrome, was not observed in our patient. Later, we performed an immunological examination discovering low CD4+ lymphocytes. That led us to use an antibiotic prophylaxis protocol with Cefotaxime, Fluconasol, and Sulfametoxazol i.v. in order to prevent superinfection.

After five days of the aggressive ventilation with PIP over $30 \mathrm{mBar}$, we decided to use pronation. Figure 4 represents Prone positioning of 12-year-old girl with critical COVID-19 pneumonia requiring artificial ventilation and complete resuscitation care.

Eighteen hours in pronation proved effective as ventilation needs quickly dropped. In the next two days, we could wean her off the artificial ventilation through SIMV (Synchronized Intermittent Mandatory Ventilation) gradually, and CPAP/PS (Continuous Positive Airway Pressure/Pressure Support) accompanied tapering the sedation. The patient was extubated on the 8th day and put on HFOT (High Flow Oxygen Therapy). Because of postintubation hypoventilation, we used a flow of $40 \mathrm{l} / \mathrm{min}$ with $\mathrm{FiO}_{2}$ : 1.0 with gradual depreciation in the next three days. We finished the ventilation support on the 13th day after intubation. Dexmedetomidine was administered to ease an opioids withdrawal syndrome.

Additionally, we terminated the Fluconazole and Sulfamethoxazole therapy because the CD4+ lymphocyte levels rose to normal. Echocardiography no longer displayed signs of decreased EF of the left ventricle. CT scan of the lung showed almost complete regression of the previous findings. (Figures 2,3) Spirometry showed a mild restriction.

The patient was discharged from the hospital on the $21^{\text {st }}$ day after intubation and the 26th day after the onset of the illness. She made a complete clinical recovery and was discharged home without supplemental oxygen. 
Dolezalova et al., Int. Ann. Sci.; Vol. 11, Issue 1, pp: 3-9, 2021

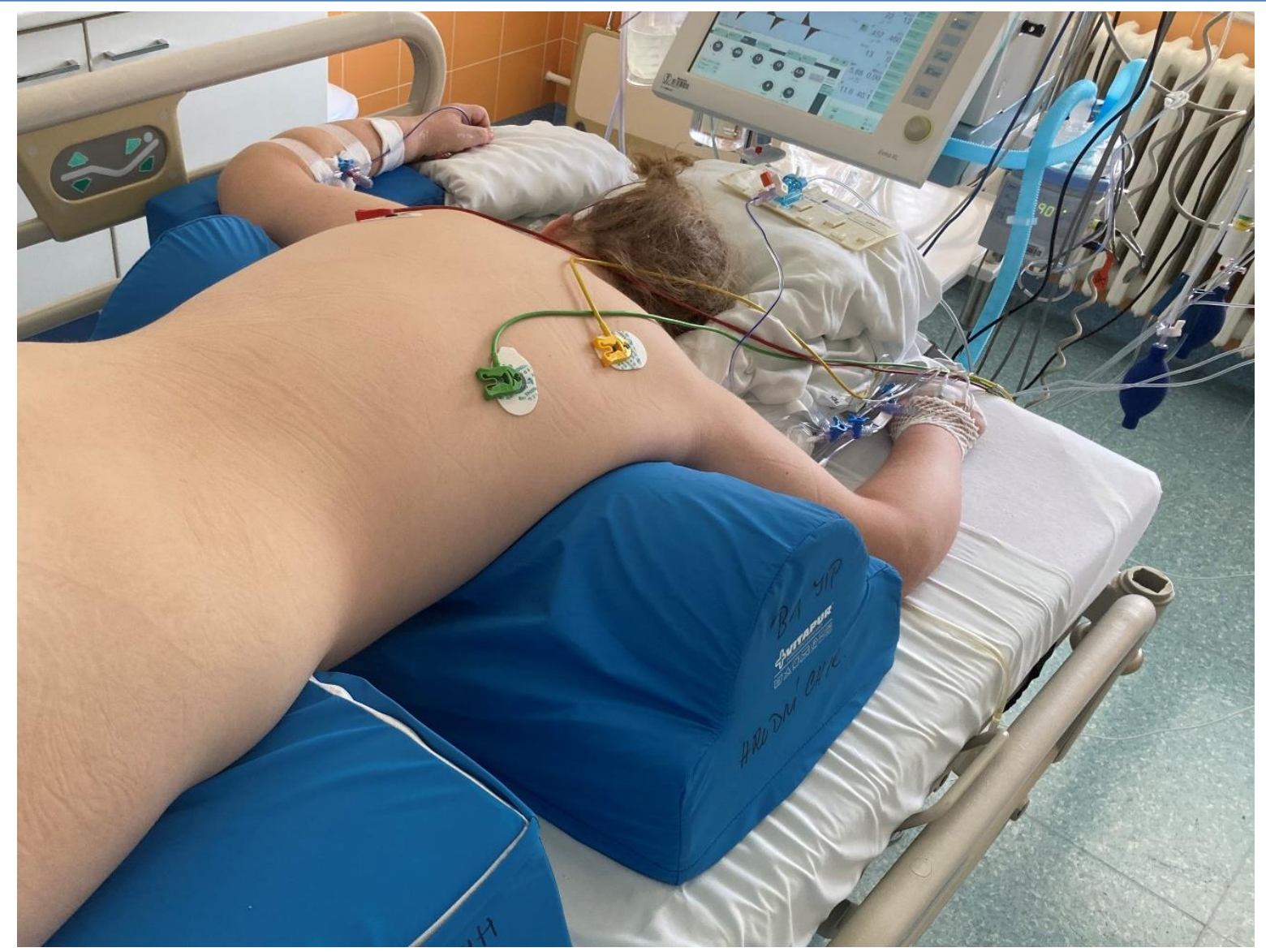

Figure 4: Prone positioning of 12-year-old girl with critical COVID-19 pneumonia requiring artificial ventilation and complete resuscitation care.

\section{Discussion}

COVID-19 in children is most likely a benign infection requiring symptomatic therapy. According to Zhou et al. [7], $70 \%$ of children have a mild illness, $13 \%$ are asymptomatic, and $7 \%$ have a moderate infection. However, in this study, no critical children were reported. In our ICU in the Paediatric Clinic of Thomayer University Hospital, we had already been trained to take intensive care of children with complications due to COVID-19 infection (MIS-C syndrome). Nevertheless, a teenage, otherwise healthy girl requiring artificial ventilation and complete resuscitation support was a surprisingly new situation unlike any other reported in the Czech Republic. The association with a critically ill child and MIS-C explains our first assumption that the acute respiratory failure and COVID positivity were another MIS-C case forcing us to start the immunoglobulin therapy. However, the laboratory findings (no elevation of CRP or PCT, no hypoalbuminemia, standard blood account), absence of mucosal changes, and a typical exanthema were not distinct for MIS-C. Inflammatory changes in the lung parenchyma can be present in both MIS-C and COVID-19 pneumonia. We settled the diagnose of COVID-19 pneumonia because of bilateral lung inflammation, which can be seen in figures 1,2,3. PCR SARS-CoV positiveness in bronchoalveolar lavage, and a short period after the onset of symptoms. The absence of GGO in CT scan of the lungs (figures 2,3), which is a typical sign of COVID-19 pneumonia in adults, can be explained by the youth of the lungs with no pre-existing changes in the lung parenchyma. On the contrary, typical bilateral consolidation and treein-bud signs were present and can be seen in presented figures 2,3.

We see no explanation for this girl having undergone such a critical form of COVID-19 pneumonia. Her unique risk factor was mild obesity. No other risk factors were detected. In this case report, we aim to demonstrate the rapid pace at which the clinical feature changed from mild symptoms to acute dyspnea and acute respiratory failure. She required an aggressive regimen of artificial ventilation and circulatory support. 
In the therapy, we successfully used remdesivir and pronation. Remdesivir is an experimental antiviral agent working as an inhibitor of the viral RNA-polymerase. Firstly, remdesivir was introduced to fight Ebola and Marburg virus infections. A double-blind, randomised, placebo-controlled trial finalized in November 2020 showed that remdesivir was superior to placebo in reducing the time to recover from COVID-19 pneumonia [8]. It can be used in patients older than 12 years with COVID-19 requiring oxygen support. In the Czech Republic, remdesivir was registered in July 2020. Prone position is adjuvant therapy for improving ventilation in patients with COVID-19 pneumonia. How to situate a patient with endotracheal intubation cannula and central venous catheter in prone position is shown in figure 4. It was introduced as a treatment method for management of ARDS patients, with good results for COVID-19 patients as well [9]. A combined antimicrobial therapy (Cefotaxime, Fluconasole and Sulfamethoxazole) was added as prevention of microbial superinfection.

CT scan repeated after fourteen days was almost physiological. In figures 2, 3 we can see a complete regression of consolidations and tree-in-bud signs, which shows a nearly magical reparatory ability of the lung parenchyma in childhood. Even though our patient is currently in a perfect health condition, pneumatological care and lung function monitoring are required.

\section{Conclusion}

Despite being rare, the critical course of COVID-19 infection in children is an existing feature. In the differential diagnosis in a COVID-19 positive child with acute respiratory and circulatory failure, we need to distinguish between acute COVID-19 infection and MIS-C. In our case, we notice a very rushed shift from mild symptoms to acute respiratory failure. Treatment of such patients should be reserved for paediatric ICU with experienced personnel. Using remdesivir and prone position in the therapy had a positive impact on the patient. In the therapy, we had a good experience with remdesivir and prone position. Contrary to adults, the complete clinical recovery was fast with full resorption of pathological changes on the CT of the thorax within fourteen days.

\section{Declarations}

\subsection{Informed Consent}

The patient and her parents consented to publish this case report and to publish the patient's image.

\subsection{Ethical Approval}

This publication was approved by Ethics Committee of Thomayer University Hospital and Institute of Clinical and Experimental Medicine. (Docket number 19391-21)

\subsection{Competing Interests}

The authors declared that no conflicts of interest exist in this publication.

\section{How to Cite this Article}

K. Dolezalova, C. Tamara, T. Hecht, and P. Heinige, "Critical COVID-19 Pneumonia with Acute Respiratory Failure in a Healthy 12-yearold Girl”, Int. Ann. Sci., vol. 11, no. 1, pp. 3-9, Sep. 2021. https://doi.org/10.21467/ias.11.1.3-9

\section{References}

[1] "Weekly epidemiological update on COVID-19 - 31 August 2021." WHO https://www.who.int/publications/m/item/weeklyepidemiological-update-on-covid-19---31-august-2021 (accessed Sep. 06, 2021).

[2] "Czech Republic (Czechia) COVID: 1,680,697 Cases and 30,406 Deaths - Worldometer." https://www.worldometers.info/coronavirus/country/czech-republic/ (accessed Sep. 06, 2021).

[3] B. M. Henry et al., "Laboratory abnormalities in children with mild and severe coronavirus disease 2019 (COVID-19): A pooled analysis and review," Clin. Biochem., vol. 81, pp. 1-8, Jul. 2020. https://doi.org/10.1016/J.CLINBIOCHEM.2020.05.012.

[4] T. H. de Souza, J. A. Nadal, R. J. N. Nogueira, R. M. Pereira, and M. B. Brandão, "Clinical manifestations of children with COVID19: A systematic review,” Pediatr. Pulmonol., vol. 55, no. 8, pp. 1892-1899, Aug. 2020. https://doi.org/10.1002/PPUL.24885.

[5] F. Palabiyik, S. O. Kokurcan, N. Hatipoglu, S. O. Cebeci, and E. Inci, "Imaging of COVID-19 pneumonia in children," The British Journal of Radiology, vol. 93, no. 1113, p. 20200647, Jul. 2020. https://doi.org/10.1259/BJR.20200647. 
Dolezalova et al., Int. Ann. Sci.; Vol. 11, Issue 1, pp: 3-9, 2021

[6] K. Dolezalova, C. Tamara, T. Hecht, and P. Heinige, "Critical COVID-19 Pneumonia with Acute Respiratory Failure in a Healthy 12-year-old Girl,” AIJR Prepr., 325, Jul. 2021. https://doi.org/10.21467/PREPRINTS.325.

[7] B. Zhou et al., "Risk profiles of severe illness in children with COVID-19: a meta-analysis of individual patients," Pediatr. Res. 2021, pp. 1-6, Mar. 2021. https://doi.org/10.1038/s41390-021-01429-2.

[8] J. H. Beigel et al., "Remdesivir for the Treatment of Covid-19 - Final Report," N Engl J Med, vol. 383, no. 19, pp. 1813-1826, May 2020. https://doi.org/10.1056/NEJMOA2007764.

[9] P. Ghelichkhani and M. Esmaeili, "Prone Position in Management of COVID-19 Patients; a Commentary," Arch. Acad. Emerg. Med., vol. 8, no. 1, pp. e48-e48, Apr. 2020. https://doi.org/10.22037/AAEM.V8I1.674.

Publish your research article in AIJR journals-

$\checkmark$ Online Submission and Tracking

$\checkmark$ Peer-Reviewed

$\checkmark$ Rapid decision

$\checkmark$ Immediate Publication after acceptance

$\checkmark$ Articles freely available online

$\checkmark \quad$ Retain full copyright of your article.

Submit your article at journals.aijr.org
Publish your books with AIJR publisher-

$\checkmark$ Publish with ISBN and DOI.

$\checkmark$ Publish Thesis/Dissertation as Monograph.

$\checkmark$ Publish Book Monograph.

$\checkmark$ Publish Edited Volume/ Book.

$\checkmark \quad$ Publish Conference Proceedings

Retain full copyright of your books.

Submit your manuscript at books.aijr.org 